\title{
Biological recovery from organic enrichment: some systems cope better than others
}

\author{
Catriona K. Macleod ${ }^{1,2, *}$, Natalie A. Moltschaniwskyj ${ }^{2}$, Christine M. Crawford ${ }^{1}$, \\ Susan E. Forbes ${ }^{1,3}$ \\ ${ }^{1}$ Marine Research Laboratories, Tasmanian Aquaculture \& Fisheries Institute, and Aquafin CRC, University of Tasmania, \\ Hobart, Tasmania 7001, Australia \\ ${ }^{2}$ School of Aquaculture, Tasmanian Aquaculture and Fisheries Institute, University of Tasmania, Launceston, \\ Tasmania 7250, Australia \\ ${ }^{3}$ Department of Environment \& Heritage, Canberra, ACT 2601, Australia
}

\begin{abstract}
This study examined the short-term recovery response at 2 salmon farms in southern Tasmania where organic loads were equivalent, but where background environmental conditions differed markedly. Although benthic communities at each of the farm locations showed good recovery over the 3 mo fallow period, community structure did not fully return to that observed under reference conditions at either location. At the Stringers Cove site the primary ecological functions of the background community were restored, but this was not the case at Creeses Mistake. These differences in recovery response were a direct reflection of background environmental conditions. Stringers Cove sediments had naturally high organic carbon content and as a result there was greater similarity in the ecological function of the unimpacted and impacted conditions at this location than at Creeses Mistake, where, under unimpacted conditions, the sediments had a very low organic content. In addition, the background fauna at Stringers Cove contained several species with reproductive strategies suited to rapid recruitment and well adapted for early recolonisation in organically enriched sediments. In contrast, the background fauna at Creeses Mistake not only changed more with the impacts of organic enrichment, but was less able to re-establish populations directly by immigration, needing to rely to a greater extent on remediation of the sediments by transitional species before being able to colonise. This has important implications for environmental management, as it suggests that the sediments in some areas have greater resilience to organic inputs.
\end{abstract}

KEY WORDS: Benthic recovery - Ecological function • Recolonisation • Organic enrichment • Resilience $\cdot$ Macrofauna $\cdot$ Salmon aquaculture

\section{INTRODUCTION}

The rate and extent of recovery of soft-sediment habitats following eutrophication events are affected by many different environmental factors, both within the impacted area and on a broader scale (Thrush \& Whitlach 2001). The nature and extent of eutrophication as well as local hydrodynamic conditions will influence recovery rate and particularly affect sediment cohesivity and faunal recruitment. Meanwhile, the background ecology will dictate the availability of colonists. Recovery is a complex process and the fac- tors affecting this are highly interrelated. However, disturbed areas recover to contain assemblages very similar to those in the adjacent ambient community (Bonsdorff 1989), so the composition of the benthic community in the surrounding areas is critical to the recovery response. A variety of physical and ecological factors influence how communities are structured: sediment grain-size, sediment stability, organic content, availability of food and trophic interactions are amongst the most important (Snelgrove \& Butman 1994), but broader environmental factors become increasingly important as the spatial scale of comparison 
increases (Whitlach \& Zajac 1985). In order to fully understand the recovery response, it is important to identify the key processes influencing broad-scale differences (Thrush \& Whitlach 2001).

Cage finfish aquaculture produces organic waste products (i.e. fish faeces, excess feed) that may result in localised organic enrichment of the sediments (Willoughby 1999). It is generally agreed that finfish cage aquaculture affects the infaunal community in the same way as other sources of organic enrichment. Many studies have been undertaken to determine the length of time required for complete recovery after fish farming, and there is considerable variability in the estimates, ranging from months (Lumb 1989, Ritz et al. 1989, Johannessen et al. 1994, Pohle et al. 2001, Pereira et al. 2004) to years (Karakassis et al. 1999, Brooks et al. 2004). However, most ongoing cage fish farming operations involve repeated organic enrichment events. Consequently, in terms of biogeochemical and infaunal response, this may have more similarity to natural organic enrichment events (e.g. fluctuations in river discharges or oceanic nutrient loads) than to other anthropogenic impacts, which tend to be either relatively constant (e.g. sewage outfalls or wood pulp mill effluent) or episodic (e.g. oil spills). There is very little information on the effects of shortterm fallowing or cage rotation on sediment recovery.

The amount of time required for sediment remediation is at present poorly understood. The rate and extent of recovery is affected by initial impact of the sediments, length of fallow period and farm location (Macleod et al. 2006). However, for ongoing farming operations to be sustainable it may not be necessary for the sediments to fully recover after each production cycle. In determining a suitable recovery level for sediments subject to recurrent impact it may be more appropriate to determine whether sediments have recovered sufficiently to be able to withstand further inputs without suffering any cumulative deterioration. If fallowing protocols fail to return sediments to such a condition, then there is a danger that sediment condition may progressively deteriorate to such an extent that ecological function is significantly impaired and farming operations may become unviable. Consequently, the sustainability of ongoing and repetitive impacts, such as fish farming, may be better assessed by establishing whether the ecological function of the system has been restored.

Most studies on the recovery of cage aquaculture operations have focussed on recovery at single sites/ leases within similar geographic areas (Ritz et al. 1989, Karakassis et al. 1999, Macleod et al. 2004, Pereira et al. 2004) and there are very few aquaculture-based studies that specifically compare the recovery responses of geographically distinct locations. Ecological conditions can have significant effects on the environmental sustainability of farming operations. Consequently, it might be expected that the sediments at different farm locations, with differing environmental conditions, would respond quite differently to similar fallowing protocols. A recent study of the regional impacts of salmon farming in Chile (encompassing 49 sites in 9 farming areas) indicated that in relation to sediment chemistry all localities were equally sensitive to farming practices, but that the ecological data (taxa richness and evenness) showed evidence of locality effects (Soto \& Norambuena 2004). Brooks et al. (2004) found significant differences in recovery rates between 2 farm leases within the same region in Canada and suggested that this may be linked to changes in local hydrology, which may affect the sedimentation processes. Similarly, in reviewing the findings of several aquaculture recovery studies, Black (2001) attributed differences in the overall estimates of recovery time to broad scale variability in the underlying environmental conditions.

Finfish farming operations in the temperate waters of Tasmania are located in a range of environments. In a recent study examining indicators of farm effects at 20 separate farm lease locations in Tasmania, Edgar et al. (2005) suggested that there were marked differences in environmental conditions and that exposure level may be a major determinant of regional variability in background ecology. A comparison of the differences in short-term recovery response, particularly changes in functional ecology, at farms with differing levels of environmental exposure would enable aquaculture and environmental managers to more accurately assess the sustainability of cage farming.

This study aimed to expand on the findings in Macleod et al. (2006) by examining broad scale differences in the ecological responses of infaunal communities to short-term periods of recovery from organic enrichment, with particular reference to the functional response. In addition, the management implications of regional variability in ecological function of the infaunal community in relation to sediment recovery were assessed.

\section{MATERIALS AND METHODS}

Study sites. Two farm locations in southeastern Tasmania_Creeses Mistake (Tasman Peninsula) and Stringers Cove (Port Esperance)-were sampled in this study (Fig. 1). These farms are broadly representative of the differing environments in which Atlantic salmon culture is undertaken in Tasmania. Creeses Mistake is a relatively exposed, shallow (20 m water depth) and fully marine site with predominantly fine 


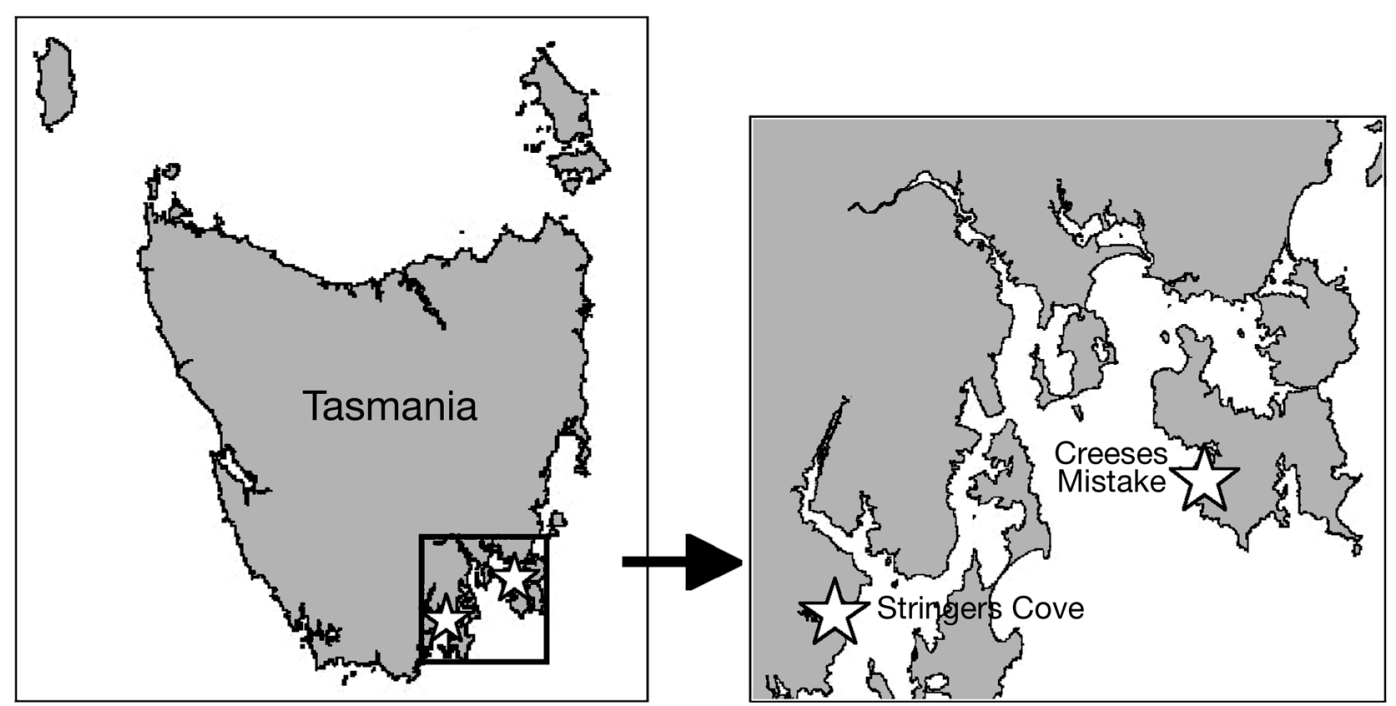

Fig. 1. Location of the 2 study farms in southeastern Tasmania: Creeses Mistake is located in Wedge Bay on the Tasman Peninsula; Stringers Cove is within Port Esperance

sand sediments, whereas Stringers Cove is in deeper $(40 \mathrm{~m})$, more sheltered waters that are occasionally subject to the freshwater influence of the nearby Esperance River with finer silt/clay sediments. Further details of site conditions and sampling positions can be found in Macleod et al. (2006).

Changes in the community structure associated with standard farm fallowing protocols were studied over 2 annual production cycles. Both farms employed an annual stocking regime where cages were stocked for 9 mo from February/March and then fallowed for 3 mo. Sediment samples were collected between February 2001 and March 2003 from cage positions and references (within the same depth range, but $150 \mathrm{~m}$ distant from the edge of selected study cages) prior to the cages being stocked (TX), at the end of 9 mo of stocking (i.e. at the start of the fallow period: T0), and at the end of a 3 mo fallow period (T3). Although the sampled cage positions had not previously been occupied, both farm leases had previously been stocked. Stocking of the leases occurred over a 4 to 6 wk period.

Faunal sampling. Five replicate sediment samples were collected from each cage position and reference using a Van Veen Grab (surface area: $0.0675 \mathrm{~m}^{2}$ ). Grab contents were transferred to mesh bags (mesh size: $0.875 \mathrm{~mm}$ ) and rinsed. Samples were wet sieved to $1 \mathrm{~mm}$ and the retained material preserved in a solution of $10 \%$ formalin:seawater. Samples were transferred to the laboratory for sorting and the infauna identified to the lowest possible taxonomic level and enumerated.

Statistical analysis. Univariate statistical analyses were used to examine spatial and temporal differences in the community structure. Analysis of variance
(ANOVA) was used to compare abundance, number of species, and Shannon diversity index both between farm locations, and within farms over the fallow period.

A similarity matrix was derived from abundance data using Bray-Curtis similarity indices and this was then used to determine the relationships between the community structures at the replicate cages and references. Abundance data were square root transformed to reduce the influence of abundant taxa and the BrayCurtis similarity index was used because of the robustness of this statistic to zero-inflated data sets (Clarke 1993). The significance of differences between conditions prior to stocking, at the reference positions and at the end of the fallow period was assessed using planned contrasts and the ANOSIM randomisation test. SIMPER analysis was used to determine the relative contribution of each taxon to the average similarity within groups and dissimilarity between groups.

Key ecological and reproductive strategies were defined for the 10 dominant species at the references and at the cage stations prior to (T0) and post- (T3) fallowing. The main ecological and life history classifications were feeding strategy, role in sediment, and reproductive strategy. Three main feeding strategies were identified: predatory carnivores (C), suspension feeders (SF) or deposit feeders (DF). However, many animals change their feeding strategies in response to environmental conditions and where this was deemed to be the case they were classified as having a variable feeding strategy (VF). The primary role that the animals play with respect to the sediments was defined as either sediment bioturbators/destabilisers (SD) or consolidators (SC). The main reproductive strategy of each species was characterised by whether adults had 
staged/seasonal (SR) or opportunistic (OR) reproductive cycles and whether larvae were benthic (B), pelagic $(\mathrm{P})$, a mix of both $(\mathrm{B} / \mathrm{P})$, or the reproductive strategy was unknown (UR). Australian marine and estuarine infauna is, on the whole, relatively poorly described, with very little species-specific biological and ecological information. Where the ecology/biology was not specifically known it was inferred using information from the next closest species or taxonomic group. Principal component analysis (PCA) of the logtransformed ecological data for the dominant species was used to examine differences in the functional strategies between cage and reference stations over time. The associated biplots show the major faunal groups, sediment and feeding role and reproductive strategies most responsible for the community changes. All multivariate analyses were conducted using the Plymouth Routines in Multivariate Ecological Research (PRIMER) software package (Clarke \& Gorley 2006).
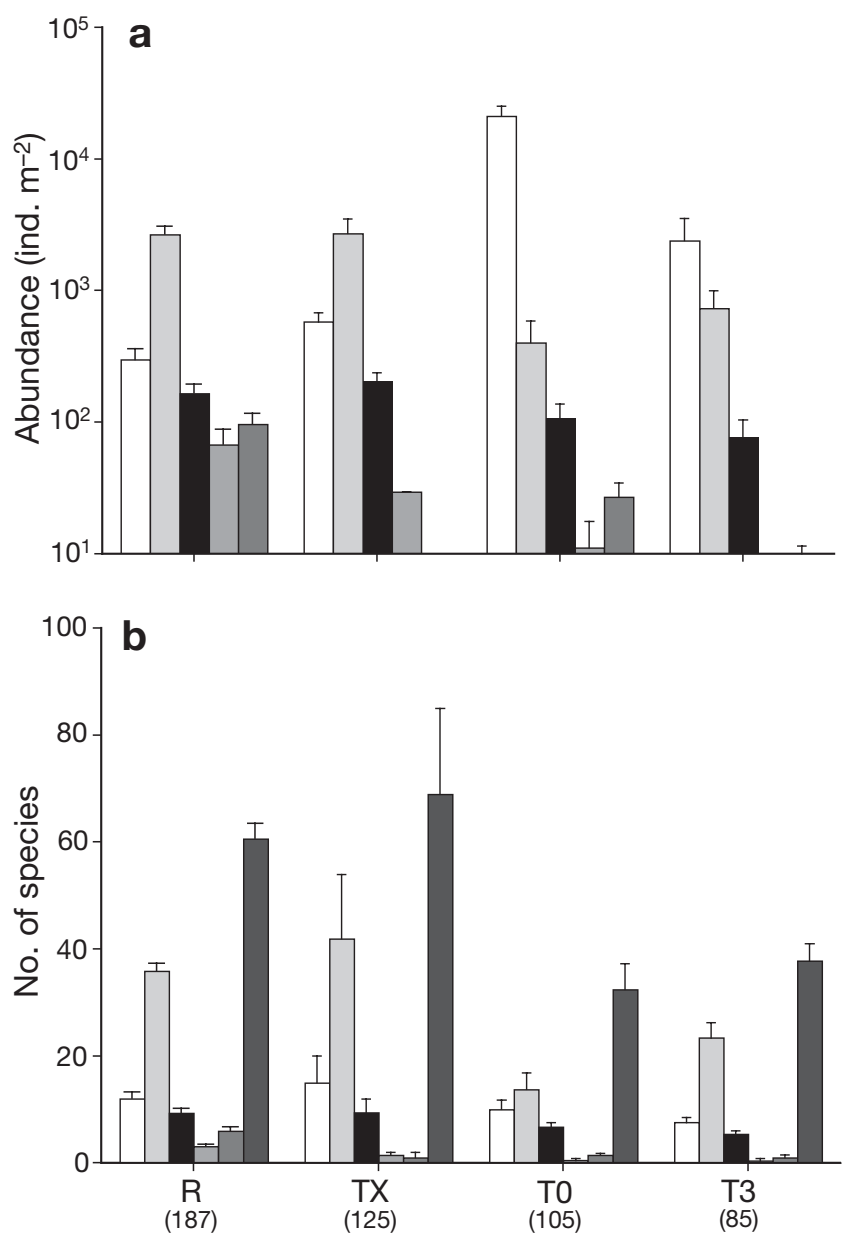

\section{RESULTS}

In a previous study (Macleod et al. 2006) significant differences were reported in the community structure of the reference and cage positions within each of the 2 farm locations and between the reference communities. The present study did not identify any numerically important species unique to either farm location, but $27 \%$ of the species were common to both farm reference sites. However, although there were similarities in species composition at both locations, the dominant taxa were markedly different (Fig. 2, Table 1).

The reference communities at Creeses Mistake had significantly more individuals and species than those at Stringers Cove (ANOVA abundance $F=17.36$, df $=1,29, \mathrm{p}<0.001$; diversity $F=38.83$, df $=1,29, \mathrm{p}<$ 0.001) (Fig. 2a,b). There was an average of $109 \pm 12$ species at Creeses Mistake compared with only $70 \pm 6$ at Stringers Cove and the abundance was $3 \times$ greater at Creeses Mistake $(4085 \pm 731)$ than at Stringers Cove
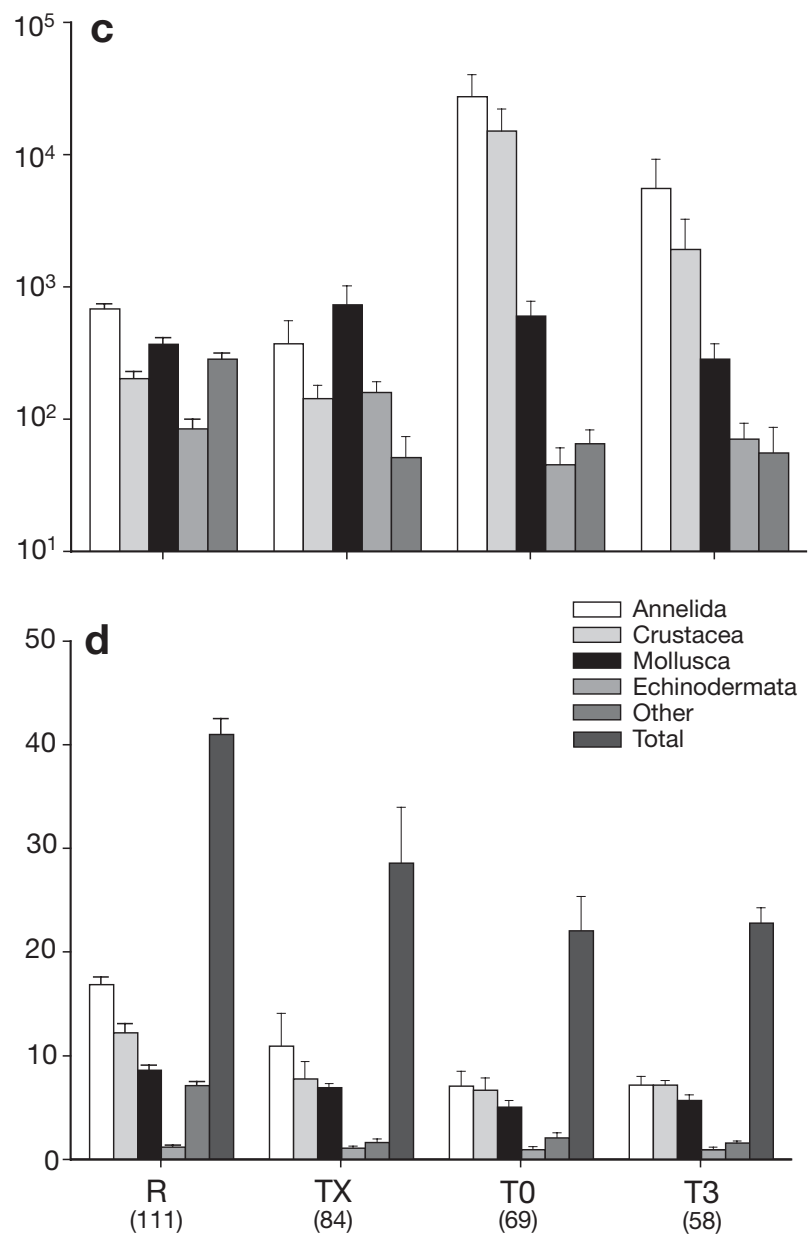

Fig. 2. $(a, c)$ Average abundance $(+\mathrm{SE})$ and $(b, d)$ number of species $(+\mathrm{SE})$ in each of the main faunal groups at $(a, b)$ Creeses Mistake and $(\mathrm{c}, \mathrm{d})$ Stringers Cove at the reference sites (R) at the start of fallowing and at the cage positions prior to stocking (TX), and at the start (T0) and end (T3) of the fallow period. Total number of species recovered indicated in parentheses beneath site labels 
$(1370 \pm 98)$. At Creeses Mistake the communities at the reference sites were characterised by a range of species from several different taxa (Table 1), but crustaceans accounted for the largest component of the fauna ( $82 \%$ of abundance, $52 \%$ of species) (Fig. 2a,b), being 8 of the top 10 dominant species (Table 1). The crustaceans Apseudes sp. 2, Ampelisca sp. 1, Euphilomedes sp. 1 and Birubius spp. were all abundant and important species characterising the Creeses Mistake reference sites (Table 1). In contrast, at Stringers Cove, annelids were the dominant taxon at the reference sites, comprising $46 \%$ of faunal abundance and $41 \%$ of species (Fig. 2c,d). At Stringers Cove the polychaete Mediomastus australiensis and the brittlestar Amphiura elandiformis were the dominant species in the reference fauna (Table 1).
After farming, the cage positions (T0) at Creeses Mistake changed markedly (Table 2), becoming dominated numerically by annelids ( $97 \%$ of fauna) and remaining this way until the end of the fallow period (T3) (Fig. 2a,b). The overall number of species at the cage positions declined from 71 to just 33, with a much greater proportional decline in the crustacean (42 to 14 species) compared to the annelid (15 to 10 species) fauna (Fig. 2b). At the start of fallowing, the polychaete Capitella capitata complex was present in excess of $17000 \mathrm{~m}^{-2}$ and this alone accounted for $73 \%$ of the overall similarity within the community (Table 1). Although it had declined at the end of the 3 mo fallow period, this species was still markedly more abundant in the post-fallowing community $\left(2621 \mathrm{~m}^{-2}\right)$ than it had been pre-stocking $\left(232 \mathrm{~m}^{-2}\right)$ (Table 1$)$.

Table 1. Relative contribution to group similarity, abundance, proportional representation within community group, ecological function and reproductive strategies of the 10 species which contributed most to overall group similarity of reference, pre-fallowing (T0) and post-fallowing (T3) communities at Creeses Mistake and Stringers Cove. Ecological function derived from literature sources. Where species-specific information was unavailable, the nearest taxonomically similar species/group has been used (results in italics). Ecological function-LG: large-bodied animal (retained on a $10 \mathrm{~mm}$ sieve); MD: medium-sized animal (retained on a $4 \mathrm{~mm}$ sieve); SM: small animal (retained on a $1 \mathrm{~mm}$ sieve); SF: suspension feeder; DF: deposit feeder; S: sediment stabilizer; D: sediment destabiliser. Reproductive strategy-MG: adults produce multiple generations; SG: adults spawn only once; P: planktonic juveniles; B: benthic juveniles; SR: staged reproductive timing (episodic/seasonal); OR: opportunistic reproductive cycle

\begin{tabular}{|c|c|c|c|c|c|c|c|}
\hline & Taxon & Phylum & $\begin{array}{l}\text { Similarity } \\
\text { contribution } \\
\qquad \%)\end{array}$ & $\begin{array}{l}\text { Abundance } \\
\text { (ind. } \mathrm{m}^{-2} \text { ) } \\
(\mathrm{SE})\end{array}$ & $\begin{array}{l}\text { Proportion } \\
\text { of total }\end{array}$ & $\begin{array}{l}\text { Ecological } \\
\text { function }\end{array}$ & $\begin{array}{l}\text { Reproductive } \\
\text { strategy }\end{array}$ \\
\hline \multicolumn{8}{|c|}{ Creeses Mistake } \\
\hline Reference & Apseudes sp. 2 & Crustacea & 11.48 & $662(250.1)$ & 20.5 & $M D, D F, D$ & $M G, B, S R$ \\
\hline (mean & Ampelisca sp. & Crustacea & 9.79 & 455 (148.4) & 14.0 & $S M, S F, S$ & $S G, B, S R(2)$ \\
\hline similarity, \%) & Euphilomedes sp. 1 & Crustacea & 7.29 & $224(45.7)$ & 6.9 & $S M, D F, D$ & Unknown \\
\hline \multirow[t]{7}{*}{$(48.85)$} & Birubius sp. & Crustacea & 6.81 & $161(29.6)$ & 5.0 & $S M, D F, D$ & $M G, B, S R$ \\
\hline & Corophium ascherusicum & Crustacea & 4.07 & $145(37.4)$ & 4.5 & $S M, D F / S F, S$ & $M G, B, S R / O R$ \\
\hline & Lysianassidae sp. 4 & Crustacea & 3.03 & $98(23.4)$ & 3.0 & $M D, C, S$ & $M G, P, S R$ \\
\hline & Echinocardium cordatum & Echinodermata & 2.92 & $73(19.88)$ & 2.3 & LG, DF, D & MG, P, SR \\
\hline & Phyllamphicteis sp. 1 & Annelida & 2.76 & $75(22.8)$ & 2.3 & $L G, S F / D F, S$ & Unknown \\
\hline & Protolembos sp. & Crustacea & 2.73 & $52(16.2)$ & 1.6 & $S M, S F / D F, S$ & $M G, B, S R$ \\
\hline & Nebalia longicornis & Crustacea & 2.71 & $36(8.5)$ & 1.1 & $M D, D F, D$ & $M G, P, O R$ \\
\hline \multirow{10}{*}{$\begin{array}{l}\text { Pre-fallowing } \\
\text { (T0) } \\
(62.84)\end{array}$} & Capitella capitata (complex) & Annelida & 73.12 & $17248(667.2)$ & 93.1 & SM, DF, D & $\mathrm{MG}, \mathrm{B} / \mathrm{P}, \mathrm{OR}$ \\
\hline & Neanthes cricognatha & Annelida & 4.24 & $199(113.9)$ & 1.1 & $M D, D F / C, D$ & $M G, B, S R$ \\
\hline & Maladnidae sp. & Annelida & 2.83 & $54(24.4)$ & 0.3 & $M D, D F, S$ & $S G, B, S R$ \\
\hline & Mysella donaciformis & Mollusca & 2.06 & $14(6.2)$ & 0.1 & $M D-L G, D F / S F, D$ & $M G, B, S R$ \\
\hline & Euphilomedes sp. 1 & Crustacea & 1.70 & $52(32.3)$ & 0.3 & $S M, D F, D$ & Unknown \\
\hline & Corbula gibba & Mollusca & 1.53 & $11(6.5)$ & 0.1 & LG, DF, D & $\mathrm{MG}, \mathrm{P}, \mathrm{SR}$ \\
\hline & Ostracoda sp. & Crustacea & 1.33 & $10(4.5)$ & 0.1 & $S M, D F, D$ & Unknown \\
\hline & Nebalia longicornis & Crustacea & 1.27 & $57(31.1)$ & 0.3 & $M D, D F, D$ & $M G, P, O R$ \\
\hline & Apseudes sp. 2 & Crustacea & 1.05 & $21(9.5)$ & 0.1 & $M D, D F, D$ & $M G, B, S R$ \\
\hline & Birubius sp. & Crustacea & 1.03 & $65(35.6)$ & 0.4 & $S M, D F, D$ & $M G, B, S R$ \\
\hline \multirow{10}{*}{$\begin{array}{l}\text { Post-fallowing } \\
\text { (T3) } \\
(35.94)\end{array}$} & Capitella capitata (complex) & Annelida & 26.38 & $2621(1343.8)$ & 74.8 & $\mathrm{SM}, \mathrm{DF}, \mathrm{D}$ & $\mathrm{MG}, \mathrm{B} / \mathrm{P}, \mathrm{OR}$ \\
\hline & Amphithoidae sp. & Crustacea & 6.78 & $37(14.6)$ & 1.1 & $S M, S F / D F, S$ & $M G, B, S R$ \\
\hline & Euphilomedes sp. 1 & Crustacea & 5.47 & $73(38.0)$ & 2.1 & $S M, D F, D$ & Unknown \\
\hline & Birubius sp. & Crustacea & 4.82 & $36(23.0)$ & 1.0 & $S M, D F, D$ & $M G, B, S R$ \\
\hline & Solemya australis & Mollusca & 4.72 & $36(19.4)$ & 1.0 & $L G, D F / S F, S$ & $M G, P$, Unknown \\
\hline & Oedicerotidae sp. & Crustacea & 4.26 & $25(9.7)$ & 0.7 & $S M, D F, D$ & $M G, B, S R$ \\
\hline & Neanthes cricognatha & Annelida & 4.00 & $94(55.5)$ & 2.7 & $M D, D F / C, D$ & $M G, B, S R$ \\
\hline & Apseudes sp. 2 & Crustacea & 3.66 & $21(9.9)$ & 0.6 & $M D, D F, D$ & $M G, B, S R$ \\
\hline & Ostracoda sp. & Crustacea & 3.45 & $67(50.8)$ & 1.9 & $S M, D F, D$ & Unknown \\
\hline & Nassarius nigellus & Mollusca & 2.57 & $22(10.0)$ & 0.6 & $M D, D F, E$ & $M G, B, S R$ \\
\hline
\end{tabular}


Table 1 (continued)

\begin{tabular}{|c|c|c|c|c|c|c|c|}
\hline & Taxon & Phylum & $\begin{array}{l}\text { Similarity } \\
\text { contribution } \\
(\%)\end{array}$ & $\begin{array}{l}\text { Abundance } \\
\text { (ind. m }{ }^{-2} \text { ) } \\
\text { (SE) }\end{array}$ & $\begin{array}{l}\text { Proportion } \\
\text { of total }\end{array}$ & $\begin{array}{l}\text { Ecological } \\
\text { function }\end{array}$ & $\begin{array}{l}\text { Reproductive } \\
\text { strategy }\end{array}$ \\
\hline $\begin{array}{l}\text { Stringers Cove } \\
\text { Reference } \\
(63.24)\end{array}$ & $\begin{array}{l}\text { Mediomastus australiensis } \\
\text { Amphiura elandiformis } \\
\text { Lysilla jennacubinae } \\
\text { Nucula pusilla } \\
\text { Thyasira adelaideana } \\
\text { Nassarius nigellus } \\
\text { Aricidea sp. } \\
\text { Nemertea sp. } 1 \\
\text { Lumbrinereis sp. } 1 \\
\text { Aschyis sp. } 2\end{array}$ & $\begin{array}{l}\text { Annelida } \\
\text { Echinodermata } \\
\text { Annelida } \\
\text { Mollusca } \\
\text { Mollusca } \\
\text { Mollusca } \\
\text { Annelida } \\
\text { Nemertea } \\
\text { Annelida } \\
\text { Annelida }\end{array}$ & $\begin{array}{r}10.17 \\
\mathrm{a} \quad 8.19 \\
5.98 \\
5.97 \\
5.01 \\
4.48 \\
4.37 \\
4.25 \\
4.07 \\
3.91\end{array}$ & $\begin{array}{l}213(32.3) \\
128(9.7) \\
75(11.0) \\
121(25.3) \\
58(4.5) \\
94(22.9) \\
75(16.3) \\
64(15.9) \\
38(5.7) \\
78(25.1)\end{array}$ & $\begin{array}{r}14.8 \\
8.9 \\
5.2 \\
8.4 \\
4.0 \\
6.5 \\
5.2 \\
4.4 \\
2.7 \\
5.4\end{array}$ & $\begin{array}{l}M D, D F, D \\
L G, D F / S F, D \\
L G, D F, S \\
L G, D F, D \\
L G, S F, D \\
M D, D F, E \\
M D, D F, D \\
M D, C / D F, D \\
L G, D F, D \\
L G, D F, S\end{array}$ & $\begin{array}{l}M G, B / P, S R \\
M G, P, S R \\
U n k n o w n \\
M G, P, S R \\
M G, B, S R \\
M G, B, S R \\
S G, P, S R \\
M G, B, S R \\
S G, B, S R \\
S G, B, S R\end{array}$ \\
\hline $\begin{array}{l}\text { Pre-fallowing } \\
\text { (T0) } \\
(39.37)\end{array}$ & $\begin{array}{l}\text { Nebalia longicornis } \\
\text { Capitella capitata (complex) } \\
\text { Corbula gibba } \\
\text { Birubius spp. } \\
\text { Nassarius nigellus } \\
\text { Neanthes cricognatha } \\
\text { Echinocardium cordatum } \\
\text { Simplisetia amphidonta } \\
\text { Nemertea sp. } 1 \\
\text { Mediomastus australiensis }\end{array}$ & $\begin{array}{l}\text { Crustacea } \\
\text { Annelida } \\
\text { Mollusca } \\
\text { Crustacea } \\
\text { Mollusca } \\
\text { Annelida } \\
\text { Echinodermata } \\
\text { Annelida } \\
\text { Nemertea } \\
\text { Annelida }\end{array}$ & $\begin{array}{r}35.85 \\
22.71 \\
7.17 \\
6.41 \\
5.53 \\
4.29 \\
3.35 \\
2.13 \\
1.54 \\
1.30\end{array}$ & $\begin{array}{l}14902(7051) \\
27470(12806) \\
353(149.2) \\
201(79.5) \\
155(87.3) \\
118(53.4) \\
43(15.4) \\
8(1.3) \\
4(4.8) \\
9(3.5)\end{array}$ & $\begin{array}{r}34.2 \\
63.1 \\
0.8 \\
0.5 \\
0.4 \\
0.3 \\
0.1 \\
<0.1 \\
<0.1 \\
<0.1\end{array}$ & $\begin{array}{l}M D, D F, D \\
\mathrm{SM}, \mathrm{DF}, \mathrm{D} \\
\mathrm{MD}-\mathrm{LG}, \mathrm{DF}, \mathrm{D} \\
S M, D F, D \\
M D, D F, E \\
S M-M D, D F / C, D \\
\mathrm{LG}, \mathrm{DF}, \mathrm{D} \\
M D, D F / C, D \\
M D, C / D F, D \\
M D, D F, D\end{array}$ & $\begin{array}{l}M G, P, O R \\
\mathrm{MG}, \mathrm{B} / \mathrm{P}, \mathrm{OR} \\
\mathrm{MG}, \mathrm{P}, \mathrm{SR} \\
M G, B, S R \\
M G, B, S R \\
M G, B, S R \\
\mathrm{MG}, \mathrm{P}, \mathrm{SR} \\
S G, P, S R \\
M G, B, S R \\
M G, B / P, S R\end{array}$ \\
\hline $\begin{array}{l}\text { Post-fallowing } \\
\text { (T3) } \\
(42.21)\end{array}$ & $\begin{array}{l}\text { Nassarius nigellus } \\
\text { Birubius spp. } \\
\text { Corbula gibba } \\
\text { Echinocardium cordatum } \\
\text { Capitella capitata (complex) } \\
\text { Theora fragilis } \\
\text { Nebalia longicornis } \\
\text { Paraprionospio coora } \\
\text { Mysella donaciformis } \\
\text { Simplisetia amphidonta }\end{array}$ & $\begin{array}{l}\text { Mollusca } \\
\text { Crustacea } \\
\text { Mollusca } \\
\text { Echinodermata } \\
\text { Annelida } \\
\text { Mollusca } \\
\text { Crustacea } \\
\text { Annelida } \\
\text { Mollusca } \\
\text { Annelida }\end{array}$ & $\begin{array}{r}19.07 \\
13.09 \\
11.66 \\
9.61 \\
8.79 \\
6.09 \\
3.76 \\
3.05 \\
3.01 \\
2.93\end{array}$ & $\begin{array}{l}201(42.0) \\
101(23.1) \\
222(77.5) \\
71(21.6) \\
5525(3685) \\
23(6.2) \\
1791(1319) \\
11(3.7) \\
11(3.7) \\
10(2.6)\end{array}$ & $\begin{array}{r}2.5 \\
1.2 \\
2.7 \\
0.9 \\
67.7 \\
0.3 \\
22.0 \\
0.1 \\
0.1 \\
0.1\end{array}$ & $\begin{array}{l}M D, D F, E \\
S M, D F, D \\
\text { MD-LG: DF: D } \\
\text { LG, DF, D } \\
\text { SM, DF, D } \\
M D, D F, D \\
M D, D F, D \\
M D, D F, D \\
M D-L G, D F / S F, D \\
M D, D F / C, D\end{array}$ & $\begin{array}{l}M G, B, S R \\
M G, B, S R \\
\mathrm{MG}, \mathrm{P}, \mathrm{SR} \\
\mathrm{MG}, \mathrm{P}, \mathrm{SR} \\
\mathrm{MG}, \mathrm{B} / \mathrm{P}, \mathrm{OR} \\
M G, P, S R \\
M G, P, \text { OR } \\
S G, P, \text { Unknown } \\
M G, B, S R \\
S G, P, S R\end{array}$ \\
\hline
\end{tabular}

Recovery over the fallow period was mainly as a result of changes in the abundances of 3 key polychaete species; Capitella capitata complex declined 10-fold, Neanthes cricognatha declined from 199 to $94 \mathrm{~m}^{-2}$ and Maldanidae sp. was absent altogether in the post-

Table 2. ANOSIM test of difference in community structure between sampling positions at Creeses Mistake and Stringers Cove (global R), including pairwise comparison of reference (R), pre-stocking (TX) and pre- (T0) and post-fallowing (T3) communities

\begin{tabular}{|llccc|} 
& \multicolumn{2}{c}{ Creeses Mistake } & \multicolumn{2}{c|}{ Stringers Cove } \\
& Rho & $\mathrm{p}$ & Rho & $\mathrm{p}$ \\
\hline Global test & $\mathbf{0 . 6 9 3}$ & $\mathbf{0 . 0 0 1}$ & $\mathbf{0 . 4 3 7}$ & $\mathbf{0 . 0 0 1}$ \\
RX vs. TX & 0.500 & 0.333 & 0.542 & 0.036 \\
R0 vs. T0 & 1.000 & 0.029 & 0.689 & 0.004 \\
R3 vs. T3 & 0.728 & 0.010 & 0.759 & 0.002 \\
T0 vs. TX & 1.000 & 0.067 & 0.467 & 0.005 \\
T0 vs. T3 & 0.425 & 0.040 & 0.230 & 0.054 \\
TX vs. T3 & 0.545 & 0.048 & 0.183 & 0.042 \\
\hline
\end{tabular}

fallowing communities (Table 3). In contrast, the proportional importance of crustaceans increased over the fallow period, and although crustaceans were still markedly less abundant after fallowing than in either the pre-stocking or reference communities (Fig. 2a,b), there was clearly some recovery in this group.

Although the total abundances at Stringers Cove were similar in the pre-stocking and reference communities (ANOVA $F=0.002$, df $=1,20, p=0.969$ ) the number of species in each of the major faunal groups was consistently and significantly lower in the prestocking communities (ANOVA $F=9.917$, df $=1,20$, $\mathrm{p}=0.005$ ) (Fig. 2c,d), possibly indicating a residual farm effect. There were significant differences in species composition between the reference, pre-stocking, pre-fallowing and post-fallowing communities (Table 2). Rho values from pairwise comparison of the data were lower for the pre-stocking comparison, indicating that the pre-stocking community (TX) was closer to the post-fallow community (T3) than to either the reference or pre-fallow communities (T0). Annelids comprised the greatest proportion of the reference 
Table 3. Differences in abundance and Bray Curtis dissimilarity level of the 10 species which most clearly distinguish between pre- (T0) and post-fallowing (T3) communities, between the pre-stocking (TX) and post-fallowing (T3) communities and between the reference and post-fallowing (T3) communities at each farm location

\begin{tabular}{|c|c|c|c|c|}
\hline \multirow{2}{*}{$\begin{array}{l}\text { Taxon } \\
\text { Creeses Mistake }\end{array}$} & \multicolumn{2}{|c|}{$\longrightarrow$ Abundance (ind. $\mathrm{m}^{-2}$ ) } & \multirow[t]{2}{*}{ Dissimilarity:SD ratio } & \multirow[t]{2}{*}{ Dissimilarity (\%) } \\
\hline & T0 & T3 & & \\
\hline Capitella capitata complex & $17248(6667)$ & $2621(1344)$ & 3.48 & 33.18 \\
\hline Neanthes cricognatha & $199(133.9)$ & $94(55.5)$ & 1.71 & 2.92 \\
\hline Maldanidae sp. 1 & $54(24.4)$ & 0 & 2.82 & 2.19 \\
\hline Spionidae sp. 1 & $6(6.2)$ & $117(71.6)$ & 0.86 & 2.03 \\
\hline Tethygeneia sp. 1 & 0 & $14(13.6)$ & 0.66 & 1.89 \\
\hline Corophium ascherusicum & $115(53.2)$ & $6(4.7)$ & 1.19 & 1.88 \\
\hline Amphithioidae sp. 1 & $10(7.0)$ & 37 (14.6) & 1.74 & 1.66 \\
\hline Nebalia longicornis & $57(31.1)$ & $23(20.3)$ & 1.28 & 1.62 \\
\hline Cumacea sp. 1 & $1(1.2)$ & $2(2.5)$ & 0.60 & 1.52 \\
\hline \multirow[t]{2}{*}{ Oedicerotidae sp. 1} & $1(1.2)$ & $25(9.7)$ & 1.77 & 1.46 \\
\hline & TX & T3 & & \\
\hline Capitella capitata complex & $232(74.8)$ & 2621 (1343.8) & 1.18 & 7.63 \\
\hline Apseudes sp. 2 & $530(507.9)$ & $21(9.9)$ & 1.08 & 5.39 \\
\hline Birubius spp. & $474(456.9)$ & $36(23.0)$ & 1.22 & 5.22 \\
\hline Spionidae sp. 2 & $122(69.9)$ & 0 & 3.72 & 3.57 \\
\hline Lyssianassidae sp. 1 & $176(126.6)$ & $6(3.1)$ & 2.54 & 3.27 \\
\hline Tethygeneia sp. 1 & $232(156.7)$ & $14(13.6)$ & 1.85 & 2.23 \\
\hline Halicarcinus ovatus & $102(92.4)$ & $2(1.4)$ & 1.2 & 2.07 \\
\hline Spionidae sp. 1 & 0 & $117(71.6)$ & 0.76 & 1.60 \\
\hline Solemya australis & $77(44.4)$ & $36(19.4)$ & 1.8 & 1.59 \\
\hline \multirow{2}{*}{ Theora fragilis } & $26(17.4)$ & 0 & 5.07 & 1.56 \\
\hline & Reference & T3 & & \\
\hline Capitella capitata complex & $5(3.5)$ & $2621(1344)$ & 1.33 & 12.39 \\
\hline Ampelisca sp. 1 & $414(255.7)$ & $5(3.5)$ & 2.17 & 4.43 \\
\hline Apseudes sp. 2 & $607(399.9)$ & $21(9.9)$ & 1.38 & 4.36 \\
\hline Spionidae sp. 1 & $1(1.23)$ & $117(71.6)$ & 1.01 & 2.21 \\
\hline Euphilomedes sp. 1 & $215(43.0)$ & $73(38.0)$ & 1.33 & 2.17 \\
\hline Lyssianassidae sp. 4 & $78(20.8)$ & $23(23.5)$ & 1.73 & 2.12 \\
\hline Photis sp. 1 & $100(59.6)$ & $4(3.7)$ & 1.81 & 2.06 \\
\hline Neanthes cricognatha & $4(2.4)$ & $94(55.5)$ & 1.05 & 2.05 \\
\hline Echinocardium cordataum & $65(32.8)$ & 0 & 2.10 & 2.02 \\
\hline Corophium ascherusicum & $102(48.9)$ & $6(4.7)$ & 1.71 & 2.02 \\
\hline \multirow{12}{*}{$\begin{array}{l}\text { Stringers Cove } \\
\text { Capitella capitata complex } \\
\text { Nebalia longicornis } \\
\text { Corbula gibba } \\
\text { Neanthes cricognatha } \\
\text { Nassarius nigellus } \\
\text { Birubius spp. } \\
\text { Theora fragilis } \\
\text { Echinocardium cordatum } \\
\text { Platyhelminthes sp. } 1 \\
\text { Mysella donaciformis }\end{array}$} & T0 & T3 & & \\
\hline & 27470 (12806) & 5525 (3685) & 1.20 & 29.13 \\
\hline & $14902(7050)$ & $1791(1320)$ & 1.58 & 25.50 \\
\hline & $353(149.2)$ & $222(77.5)$ & 1.30 & 3.40 \\
\hline & $118(53.4)$ & $4(1.6)$ & 0.95 & 2.94 \\
\hline & $155(87.3)$ & $201(42.0)$ & 1.19 & 2.82 \\
\hline & $201(79.5)$ & $101(23.1)$ & 1.38 & 2.43 \\
\hline & $53(45.0)$ & $23(6.2)$ & 0.97 & 1.91 \\
\hline & $43(15.4)$ & $71(21.6)$ & 1.32 & 1.67 \\
\hline & $30(15.7)$ & 0 & 1.06 & 1.44 \\
\hline & $20(10.5)$ & $11(3.7)$ & 1.28 & 1.33 \\
\hline & TX & T3 & & \\
\hline Capitella capitata complex & $2(1.1)$ & 5525 (3685) & 0.75 & 14.97 \\
\hline Nebalia longicornis & $2(1.0)$ & 1791 (1320) & 0.66 & 7.55 \\
\hline Corbula gibba & $515(232.8)$ & $222(77.5)$ & 1.07 & 7.04 \\
\hline Echinocardium cordatum & $112(47.9)$ & $71(21.6)$ & 1.80 & 3.48 \\
\hline Nassarius nigellus & $151(56.0)$ & $201(42.0)$ & 1.74 & 3.29 \\
\hline Mediomastus australiensis & $89(50.9)$ & $2(1.3)$ & 1.13 & 2.94 \\
\hline Birubius spp. & $40(16.0)$ & $101(23.1)$ & 1.29 & 2.82 \\
\hline Nemertea sp. 1 & $36(23.0)$ & $9(4.4)$ & 1.01 & 1.94 \\
\hline Aricidea sp. 1 & $66(42.9)$ & 0 & 0.68 & 1.94 \\
\hline \multirow[t]{2}{*}{ Nemertea sp. 2} & $9(7.3)$ & $36(32.4)$ & 0.77 & 1.92 \\
\hline & Reference & T3 & & \\
\hline Capitella capitata complex & $19(5.1)$ & $5525(3685)$ & 0.75 & 10.38 \\
\hline Nebalia longicornis & 0 & $1791(1320)$ & 0.66 & 5.49 \\
\hline Mediomastus australiensis & $237(51.8)$ & $2(1.3)$ & 4.40 & 5.02 \\
\hline Nucula pusilla & $180(23.9)$ & $2(1.6)$ & 3.28 & 4.52 \\
\hline Amphiura elandiformis & $138(10.1)$ & $1(0.6)$ & 4.43 & 4.06 \\
\hline Lysilla jennacubinae & 79 (14.5) & 0 & 4.35 & 3.08 \\
\hline Echinocardium cordatum & 0 & $71(21.6)$ & 1.52 & 2.90 \\
\hline Thyasira adelaideana & $63(8.2)$ & 0 & 3.98 & 2.80 \\
\hline Birubius spp. & $11(5.5)$ & $101(23.1)$ & 1.63 & 2.60 \\
\hline Corbula gibba & $33(7.9)$ & $222(77.5)$ & 1.28 & 2.43 \\
\hline
\end{tabular}


community ( $37 \%$ of total abundance, $53 \%$ of species; Fig. 2c,d). The pre-stocking community still had proportionally many more species of annelid than any other taxonomic group (44\%), but the abundance of molluscs had increased markedly (Fig. 2c), mostly due to increases in the abundance of Corbula gibba (from 37 to $515 \mathrm{~m}^{-2}$ ) and Nassarius nigellus ( 89 to $151 \mathrm{~m}^{-2}$ ) at the pre-stocking positions.

At the start of fallowing (T0) the abundance of the 2 known opportunists, Capitella capitata complex and Nebalia longicornis, had increased markedly (Table 1). The large increase in abundance of these 2 species was the primary cause of the significant increase in annelids (ANOVA $F=4.301, \mathrm{df}=1,22, \mathrm{p}=0.01$ ) and crustaceans (ANOVA $F=4.301, \mathrm{df}=1,22, \mathrm{p}=0.005$ ) relative to the references at this time (Fig. 2c). At the end of the fallow period (T3) the community structure remained significantly different from the reference (Table 2). Although several molluscs, particularly the scavenging gastropod Nassarius nigellus and the introduced bivalves Corbula gibba and Theora fragilis, were important features of the fallowed communities (Table 2), the most important species change over the fallow period was the decline in abundance of the opportunistic species Capitella capitata complex and Nebalia longicornis (Table 3).

Table 4. Proportional contribution of key ecological functions and reproductive strategies for the 10 species which contributed most to the overall group similarity at the background (Reference) and post-fallowing communities (T3) communities

\begin{tabular}{|lrrrr|}
\hline \multirow{2}{*}{ Function } & \multicolumn{2}{c}{ Creeses Mistake } & \multicolumn{2}{c|}{ Stringers Cove } \\
& Reference & T3 & Reference & T3 \\
\hline Size & & & & \\
Small & 58 & 97 & 7 & 74 \\
Medium & 34 & 1 & 73 & 26 \\
Large & 8 & 1 & 20 & 0 \\
Feeding strategy & & & & \\
Suspension feeding & 22 & 0 & 6 & 0 \\
Deposit feeding & 59 & 95 & 74 & 100 \\
Carnivore & 5 & 0 & 0 & 0 \\
Variable feeding & 14 & 5 & 20 & 0 \\
Sediment role & & & & \\
Epibenthic & 0 & 1 & 0 & 2 \\
Stabilising & 41 & 2 & 16 & 0 \\
Destabilising & 59 & 97 & 74 & 98 \\
Unknown & 0 & 0 & 0 & 0 \\
Reproductive strategy & & & & \\
Staged & 39 & 5 & 39 & 4 \\
Opportunistic & 20 & 89 & 20 & 92 \\
Unknown & 41 & 6 & 41 & 4 \\
Larval development & & & & \\
Pelagic & 11 & 1 & 11 & 27 \\
Benthic & 73 & 8 & 73 & 3 \\
Benthic/Pelagic & 0 & 86 & 0 & 70 \\
Unknown & 16 & 5 & 16 & 0 \\
\hline
\end{tabular}

The post-fallowing community (T3) at Stringers Cove was very different to the reference communities, but was closer to the pre-stocking community (Table 2). The main differences between the post-fallow community and both the reference and the prestocking communities were the marked reductions in abundance of Capitella capitata complex and Nebalia longicornis (Table 3). In the pre-fallowing community these 2 species accounted for $59 \%$ of the overall community similarity, but this was reduced to only $13 \%$ in the post-fallow community and neither species registered in the top 10 species in the reference communities (Table 1).

There were a range of functional and reproductive strategies in the communities at both farm locations, but, as with the species composition, there were some clear differences between the farms. Although deposit feeding was the dominant feeding strategy at both farm sites, there were proportionally more suspension feeders in the reference fauna at Creeses Mistake (Table 4). After farming there was a major change in the functional ecology of the dominant species at the cage positions (Fig. 3). The group errant polychaetes most closely reflected farming effects (Fig. 3). Postfarming these relatively mobile polychaetes, with an opportunistic reproductive strategy (i.e. Capitella capitata complex), dominated at both farm locations (Figs. $3 \& 4 \mathrm{a}-\mathrm{c}$ ). At Stringers Cove there was an increase in crustaceans (Fig. 2), primarily due to increases in abundance of the opportunist, Nebalia longicornis (Table 1). Over the fallow period, these opportunistic species markedly decreased in abundance at both farms (Table 3).

At Creeses Mistake there was very little difference in the community function at the reference sites over time. The greatest changes in functional and ecological response occurred at the cage stations over the fallow period. PCA shows that $95 \%$ of the variability in the sediment role and the feeding and reproductive strategies was associated with $\mathrm{PC} 1$, which reflects the separation of the farm stations over the fallow period (Fig. 4). At the start of fallowing (T0) the community at the cage stations was strongly dominated by deposit feeding sediment destabilisers/ bioturbators, and this remained the case until the end of the fallow period (Fig. $4 \mathrm{a}-\mathrm{c}$ ). In the post-fallowing communities the overall reproductive strategy did not change greatly, with opportunistic life strategies still proportionately more evi- 

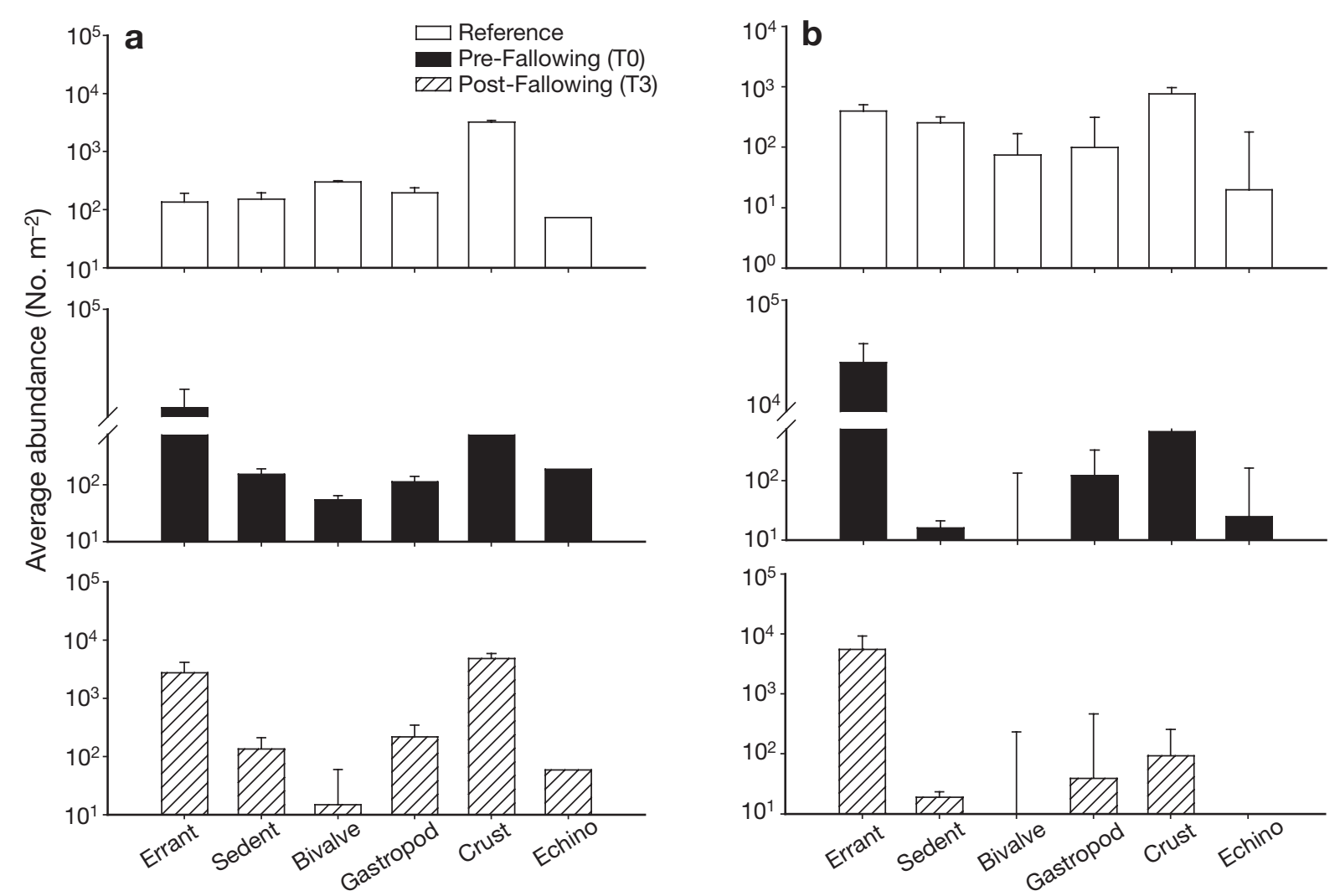

Fig. 3. Average abundance (+SE) of faunal groups represented by 10 dominant species at (a) Creeses Mistake and (b) Stringers Cove. Errant and Sedent: errant and sedentary polychaetes, respectively; Crust: crustacean; Echino: echinoderm

dent than at the reference sites. At the end of the 3 mo fallow period there was an increase in abundance of species for which the ecology is less well known (i.e. where the sediment interaction and reproductive strategy were undefined) but the proportion of opportunistic sediment bioturbators/destabilisers remained high.

At Stringers Cove the main functional differences were once again associated with the cage stations, PC1 accounting for more than $96 \%$ of the overall variability in sediment role, feeding and reproductive strategies (Fig. $4 \mathrm{~d}-\mathrm{f}$ ). There was a clear gradient of change in function between the reference, post- and prefallowing communities (Fig. $4 \mathrm{~d}-\mathrm{f}$ ). The greatest functional change was between the reference and the prefallowing stations, where there was a marked increase in the proportion of deposit feeders and sediment destabilisers in the pre-fallowing communities. There was also a change in reproductive strategy, to a community dominated by opportunistic species (Fig. $4 \mathrm{~d}-\mathrm{f}$ ). After fallowing (T3), there was some re-establishment of the fauna present at the reference sites, but the suspension feeders and sediment stabilisers had not returned and the fauna still contained a large proportion of opportunistic species (Table 4, Fig. 4d-f). The main functional differences between the pre- and postfallowing communities were due to changes in repro- ductive strategy; the dominant species in the postfallowing community tended towards benthic larval reproduction rather than pelagic larvae, which was the case in the reference communities (Fig. 4d-f).

\section{DISCUSSION}

There were marked differences in the recovery response at each of the 2 farm locations. There were changes in the infaunal community structure over the fallow period, but 3 mo was not sufficient to restore the background community structure at either location. Ecosystem theory suggests that the ecosystem is a complex and stable self-regulating system which has evolved mechanisms for self-repair (O'Neil 1999). Populations within an ecosystem are adapted to resist and recover from random fluctuations in the environment (Odum 1969). However, there is also a view that if subjected to sufficient disturbance ecosystems can jump to a new configuration where, although recovery may still occur, it does not return to the original ecosystem (Holling 1973, Young et al. 2001). In the present study the pattern of recovery at both locations was broadly consistent with the classical organic enrichment response model described by Pearson \& Rosenberg (1978), 
albeit with locally specific indicators of the various successional stages (Fig. 5). The impacted communities were very similar in both systems, characterised by pollutant-tolerant opportunists, in this instance Capitella capitata, a small deposit-feeding polychaete indicative of organic enrichment (Grassle \& Grassle 1974, Pearson \& Rosenberg 1978). At both locations there was a progressional change in the community structure over the recov- ery period. However, there were major differences in the recovery response primarily as a result of the background conditions and resultant differences in the communities at each of the farm locations.

In this study the structure of the background communities reflected the individual characteristics of each environment. Creeses Mistake is fully marine and relatively exposed with predominantly fine sand sediments
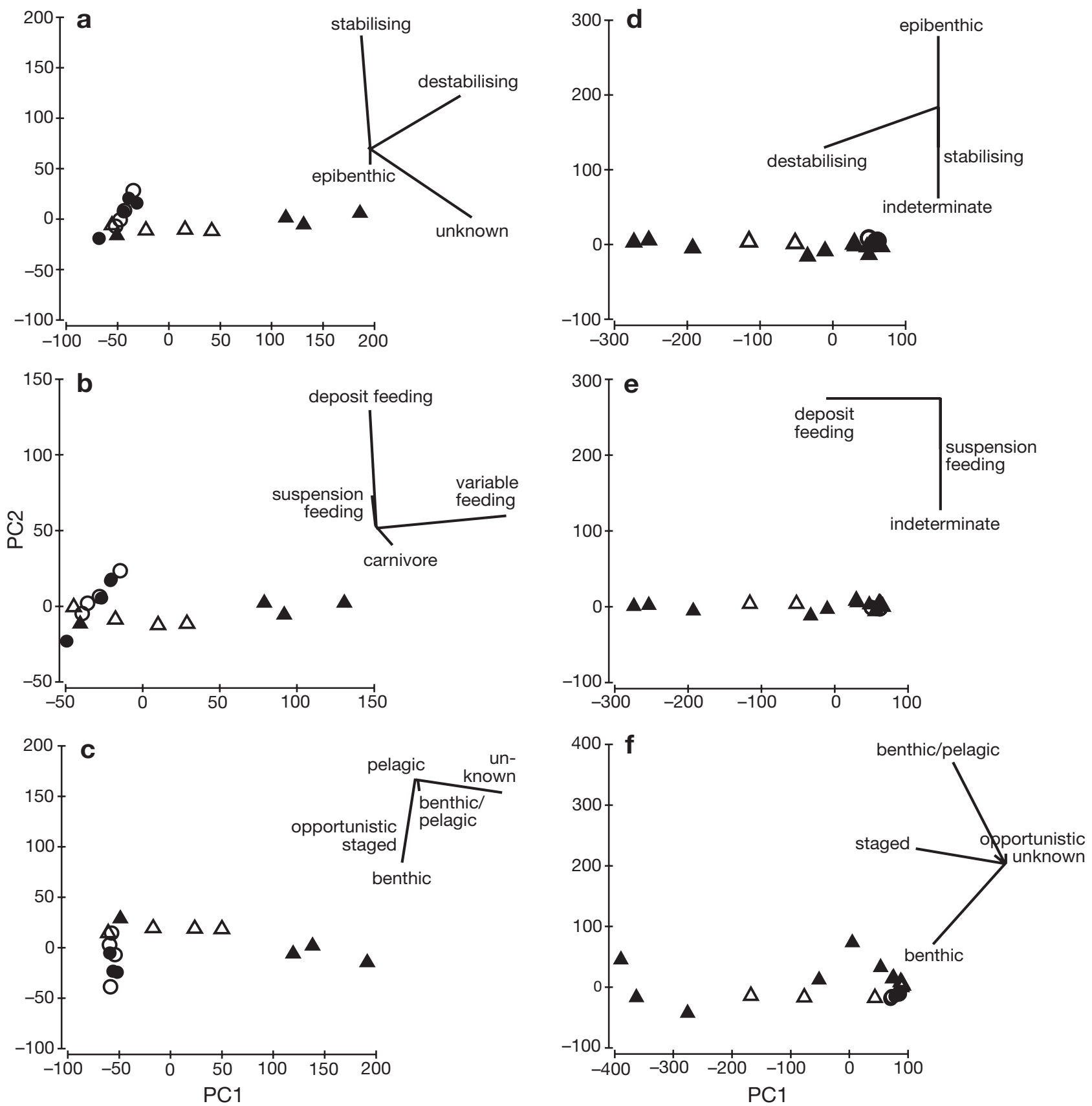

Fig. 4. Principal component analysis with biplot showing $(\mathrm{a}, \mathrm{d})$ sediment role, $(\mathrm{b}, \mathrm{e})$ feeding strategy, $(\mathrm{c}, \mathrm{f})$ reproductive strategy most strongly associated with site separations of reference $(\bullet, T 0 ; \mathrm{O}, \mathrm{T} 3)$ and pre- $(\boldsymbol{\Delta}, \mathrm{T} 0)$ and post- $(\Delta, \mathrm{T} 3)$ fallowing stations at $(\mathrm{a}-\mathrm{c})$ Creeses Mistake and $(\mathrm{d}-\mathrm{f})$ Stringers Cove based on information for top 10 species in each group. In all cases principal components 1 and 2 (PC1 and PC2) account for $>99 \%$ of total variance 


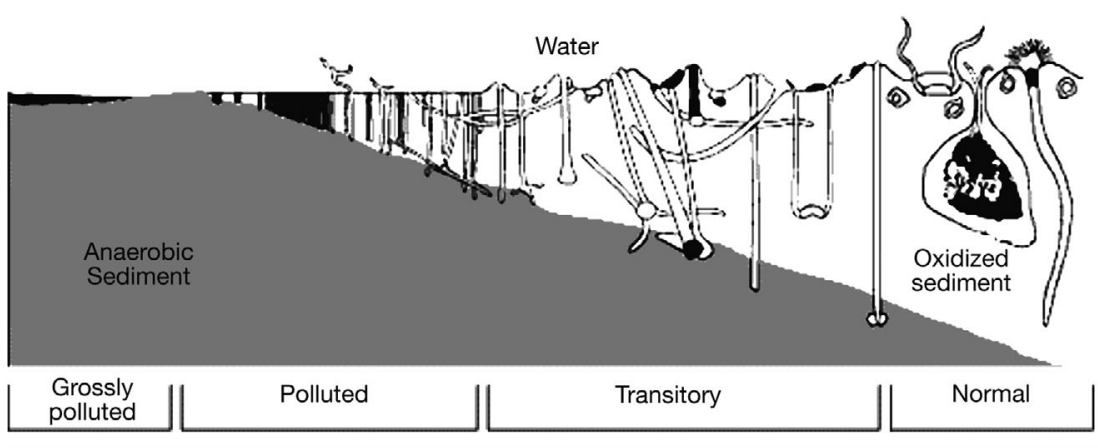

Dominant genera in Pearson \& Rosenberg model (1978)

$\begin{array}{lllll}\text { No Fauna } & \text { Capitella } & \text { Labidoplax } & \text { Chaetozone } & \text { Nucula } \\ & \text { Scolelepis } & \text { Corbula } & \text { Anatides } & \text { Amphiura } \\ & & \text { Goniada } & \text { Pectinaria } & \text { Terebellides } \\ & \text { Thyasira } & \text { Myriochele } & \text { Rhodine } \\ & \text { Pholoe } & \text { Ophiodromus } & \text { Echinocardium } \\ & & & & \text { Nephrops }\end{array}$

Dominant taxa in current study

Creeses Mistake

N/A Capitella capitata

Stringers Cove

N/A Capitella capitata
Spionidae sp. 2

Neanthes cricognatha

Nassarius nigellus

Theora fragilis

Corbula gibba

(Echinocardium cordatum)
Apseudes sp. 2 Ampelisca sp.

Amphiura elandiformis Mediomastus australiensis (Lysilla jennacubinae) (Thyasira adelaideana)

son \& Rosenberg (1978) ccession model showing local indicator taxa

whereas the Stringers Cove site is more sheltered with silt-clay sediments (Macleod et al. 2004). In general, suspension feeders tend to be most abundant in high energy environments whilst deposit-feeders are more abundant in depositional areas with fine-grained muddy sediments (Snelgrove 1999). Consequently, the ecology of the unimpacted communities was specifically and functionally quite different at each farm location; bioturbating and deposit feeding annelids were the predominant faunal group at Stringers Cove whilst Creeses Mistake had a greater proportion of suspension feeders and sediment stabilisers. The resilience of the system, defined as the rate at which measured responses attain pre-disturbance levels (Power 1999), will vary with the type and magnitude of disturbance (Gore et al. 1990). The biological attributes and ecology of the surrounding area play a significant role in the recovery response (Niemi et al. 1990).

Although community composition at Stringers Cove altered markedly after farming, the unimpacted and impacted communities were functionally quite similar. The functional significance of changes in the community composition depends on the species in question; some species may be lost without substantially altering the system function, whilst the loss of others may have serious consequences (Snelgrove 1999). The dominant species at the reference positions at Stringers Cove were the brittle star Amphiura elandiformis and the polychaete Mediomastus australiensis, both of which are active bioturbators (Rosenberg et al. 1997). Mediomastus species are also often found where organic content has been slightly enhanced (Levin 2000). This suggests that the background fauna at Stringers Cove was adapted to unconsolidated sediments with increased organic content.

In contrast, the background community at Creeses Mistake was strongly characterised by 2 suspensionfeeding crustacean species, the tanaid Apseudes sp. 2 and the ampeliscid amphipod Ampelisca sp., both of which are tube builders which would consolidate the sediments. When environmental conditions deteriorate crustaceans are often amongst the first members of the infauna to be affected (Nilsson \& Rosenberg 1994). High levels of organic enrichment resulting from farming would overwhelm and eliminate this suspensionfeeding community and recolonisation of these species would be constrained by the physical actions of a large deposit-feeding population, which would both undermine sediment cohesivity and clog filtering structures through sediment resuspension (Rhoads \& Young 1970). Accordingly, the background community at Creeses Mistake would have much greater difficulty in re-establishing itself than that of Stringers Cove. Consequently, the community would be unlikely to exhibit functional recovery until the recovery process was well underway. 
For community structure to fully recover, sediment conditions must be able to support the background fauna. Recovery will be slower in areas where the background fauna is unable to recolonise, either because the conditions are unsuitable for larval settlement or species immigration or because the reproductive cycles of the local species do not correspond to the recovery timeframe. Early colonisers play a critical role in ameliorating sediment biogeochemical conditions for subsequent species (Rhoads 1974). Habitat condition plays an important role in determining settlement success and organic content is a specific sediment cue for many species (Butman et al. 1988). The background organic carbon levels at Stringers Cove were relatively high (ca. 3\%) (Macleod et al. 2006) and consequently the background fauna at Stringers Cove would be pre-adapted to sediments with high levels of organic material. Although many of these species may have been overwhelmed by the additional organic loadings associated with farming activities, once farming ceased the environmental conditions would not be as inhospitable to these species and they would recolonise relatively easily. The reproductive strategies of the native fauna at Stringers Cove were also well suited to rapid recolonisation, with a large proportion of the dominant species able to produce multiple generations of benthic larvae which could migrate directly to the recovering sediments from adjacent areas.

In contrast, the background organic carbon levels at Creeses Mistake were very low $(0.2 \%)$, reflecting the sandy nature of this site (Macleod et al. 2004). After farming, the sedimentary carbon loading at Creeses Mistake had increased 5-fold (Macleod et al. 2004). The results indicate that after farming, the background suspension-feeding community had been largely eliminated and that the feeding ecology had shifted to a community dominated by deposit feeders. Although species utilising different trophic modes can co-occur in large numbers, and distributions of suspension- and deposit-feeders are not mutually exclusive (Snelgrove \& Butman 1994), bioturbation and sediment resuspension associated with large numbers of sediment deposit-feeders will often inhibit suspension-feeding communities (Rhoads \& Young 1970, Brenchley 1981), and this may explain the elimination of the key stabilising species in the background community observed at Creeses Mistake. It also suggests that for the background community to re-establish at Creeses Mistake there would need to be a significant reduction in both the accumulated organic material and the abundance of bioturbating deposit-feeders. In addition, several of the important species in the background communities at Creeses Mistake had staged reproductive strategies and so would only be repro- ductive at specific times of the year, thus slowing the recovery response by limiting the supply of larvae available for recolonisation.

The background fauna at Creeses Mistake was more diverse than at Stringers Cove, with almost $70 \%$ more species overall recovered at Creeses Mistake. Areas with diverse communities tend to have a wider range of ecological functions, including species' mobility and reproductive strategies, and such communities will take longer to recover than those where diversity is low and the communities are simple (Thrush \& Whitlach 2001). Consequently, impacts will be more significant in areas, such as Creeses Mistake, with inherently high diversity (Thrush \& Whitlach 2001).

These findings have interesting implications for locating and managing cage aquaculture operations specifically, but also for other more general organic enrichment sources. The findings further substantiate the conclusions drawn by Macleod et al. (2006), who suggest that the premise that it is better for the environment to locate farms in more exposed locations to reduce the impact of organic enrichment by spreading the effects may be flawed. This study shows that, under similar farming impacts, there was a greater change in the benthic infaunal community and ecosystem function at the more exposed location than at the more sheltered location and that the recovery response was slower. In addition, the overall area affected by organic deposition will be greater at exposed locations compared with more sheltered sites because the current flow and or tidal influences are greater, thus increasing the field of dispersal. The fauna at more sheltered locations where organic-rich sediments accumulate may actually have a natural resilience to organic loading, being ecologically and functionally pre-adapted to cope with an increased level of organic enrichment. This suggests that, so long as the carrying capacity of system is not exceeded, sheltered locations may in fact be better suited to caged fish-farming. Finally, the differences in the recovery time with location further reinforce the contention that managing recovery should take into account features of the receiving environment such as sediment type, organic matter content and ecological function of the resident infauna.

Acknowledgements. This work formed part of a project of the Co-operative Research Centre for Sustainable Aquaculture of Finfish (Aquafin CRC) and received funds from the Australian Government's CRC Program, the Fisheries Research and Development Corporation (FRDC) and other CRC participants. The authors acknowledge the support of the Tasmanian Aquaculture Industry, both collectively through the Tasmanian Salmon Growers Association, as well as the individual support of Tassal Ltd, and the field and laboratory assistance of B. Connell, S. Dickson and I. M. Mitchell. 


\section{LITERATURE CITED}

Black KD (2001) Environmental impacts of aquaculture. Sheffield Academic Press, Sheffield

Bonsdorff E (1989) Infaunal colonization and its dependence on environmental variation-experimental evidence from the northern Baltic Sea. In: Ryland JS, Tyler PA (eds) Reproduction, genetics and distribution of marine organisms. 23rd Euro Mar Biol Symp. Olsen \& Olsen, Fredensborg, p 349-356

Brenchley GA (1981) Disturbance and community structure: an experimental study of bioturbation in marine softbottom environments. J Mar Res 39:767-790

Brooks KM, Stierns AR, Backman C (2004) Seven year remediation study at the Carrie Bay Atlantic salmon (Salmo salar) farm in the Broughton Archipelago, British Columbia, Canada. Aquaculture 239:81-123

Butman CA, Grassle JP, Webb JM (1988) Substrate choices made by marine larvae settling in still water and in a flume flow. Nature 333:771-773

Clarke KR (1993) Non-parametric multivariate analyses of changes in community structure. Aust J Ecol 18:117-143

Clarke KR, Gorley RN (2006) PRIMER V.6: user manual/tutorial. Primer-E, Plymouth

Edgar GJ, Macleod CK, Mawbey RB, Shields D (2005) Broadscale effects of marine salmonid aquaculture on macrobenthos and the sediment environment in southeastern Tasmania. J Exp Mar Biol Ecol 327:70-90

Gore JA, Kelly JR, Yount JD (1990) Application of ecological theory to determining recovery potential of disturbed lotic ecosystems: research needs and priorities. Environ Manage 14:755-762

Grassle JF, Grassle JP (1974) Opportunistic life histories and genetic systems in marine benthic polychaetes. J Mar Res 32:253-284

Holling CS (1973) Resilience and stability of ecological systems. Annu Rev Ecol Syst 4:1-23

Johannessen PJ, Botnen HB, Tvedten OF (1994) Macrobenthos: before, during and after a fish farm. Aquacult Fish Manage 25:55-66

Karakassis I, Hatziyanni E, Tsapakis M, Plaiti W (1999) Benthic recovery following cessation of fish farming: a series of successes and catastrophes. Mar Ecol Prog Ser 184:205-218

Levin LA (2000) Polychaetes as environmental indicators: response to low oxygen and organic enrichment. Bull Mar Sci 67:668-678

Lumb CM (1989) Self-pollution by Scottish salmon farms? Mar Pollut Bull 20:375-379

Macleod C, Bissett A, Burke C, Forbes S, Holdsworth D, Nichols P, Revill A, Volkman J (2004) Development of novel methods for the assessment of sediment condition and determination of protocols for sustainable finfish cage aquaculture operations. Aquafin CRC Project 4.1 - Final Report. Tasmanian Aquaculture and Fisheries Institute, University of Tasmania, Hobart

Editorial responsibility: Howard Browman (Associate Editorin-Chief), Storebø, Norway
Macleod CK, Moltschaniwskyj NA, Crawford CM (2006) Evaluation of short-term fallowing as a strategy for the management of recurring organic enrichment under salmon cages. Mar Pollut Bull 52:1458-1466

Neimi GJ, DeVore PW, Detenbeck NE (1990) Overview of case studies on recovery of aquatic ecosystems from disturbance. Environ Manage 14:571-588

Nilsson HC, Rosenberg R (1994) Hypoxic response of two marine benthic communities. Mar Ecol Prog Ser 115: 209-217

Odum EP (1969) The strategy of ecosystem development. Science 164:262-270

O'Neill RV (1999). Recovery in complex ecosystems. J Aquat Ecosyst Stress Recov 6:181-187

Pearson TH, Rosenberg R (1978) Macrobenthic succession in relation to organic enrichment and pollution of the marine environment. Oceanogr Mar Biol Annu Rev 16:229-311

Pereira PMF, Black KD, McLusky DS, Nickell TD (2004) Recovery of sediments after cessation of marine fish farm production. Aquaculture 235:315-330

Pohle G, Frost B, Findlay R (2001) Assessment of regional benthic impact of salmon mariculture within the Letang Inlet, Bay of Fundy. ICES J Mar Sci 58:417-426

Power M (1999) Recovery in aquatic ecoystems: an overview of knowledge and needs. J Aquat Ecosyst Stress Recov 6: 253-257

Rhoads DC (1974) Organism-sediment relations on the muddy seafloor. Oceanogr Mar Biol Annu Rev 12:263-300

Rhoads DC, Young DK (1970) The influence of deposit-feeding organisms on sediment stability and community trophic structure. J Mar Res 28:150-178

Ritz DA, Lewis ME, Ma Shen (1989) Response to organic enrichment of infaunal macrobenthic communities under salmonid seacages. Mar Biol 103:211-214

Rosenberg R, Nilsson HC, Hollertz K, Hellman B (1997) Density-dependent migration in an Amphiura filiformis (Amphiuridae, Echinodermata) infaunal population. Mar Ecol Prog Ser 159:121-131

Snelgrove PVR (1999) Getting to the bottom of marine biodiversity: sedimentary habitats. Bioscience 49:129-138

Snelgrove PVR, Butman CA (1994) Animal-sediment relationships revisited: cause versus effect. Oceanogr Mar Biol Annu Rev 32:111-177

Thrush SF, Whitlach RB (2001) Recovery dynamics in benthic communities: balancing detail with simplification. In: Reise K (ed) Ecological comparisons of sedimentary shores. Springer-Verlag, Berlin, p 297-311

Whitlach RB, Zajac RN (1985) Biotic interactions among estuarine infaunal opportunistic species. Mar Ecol Prog Ser 21:299-311

Willoughby S (1999) Manual of salmonid farming. Fishing News Books, Blackwell Science, Oxford

Young TP, Chase JM, Huddleston RT (2001) Succession and assembly as conceptual bases in community ecology and ecological restoration. Ecol Restor 19:5-19

Submitted: May 22, 2006; Accepted: January 11, 2007

Proofs received from author(s): July 9, 2007 